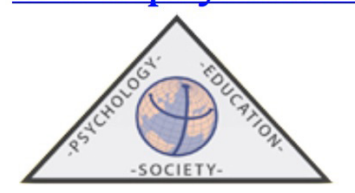

\title{
Realidad aumentada (RA) y procesos didácticos en la universidad: estudio descriptivo de nuevas aplicaciones para el desarrollo de competencias digitales
}

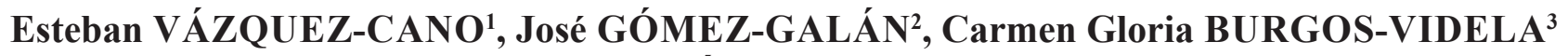 \\ y Eloy LÓPEZ-MENESES ${ }^{4}$
}

\author{
(1)Universidad Nacional de Educación a Distancia (UNED) \\ (2)Universidad de Extremadura / Universidad Ana G. Méndez \\ (E-mail: jgomez@unex.es / jogomez@suagm.edu \\ (3)Universidad de Atacama (E-mail: carmen.burgos@uda.cl) \\ (4)Universidad Pablo de Olavide (E-mail: elopmen@upo.es)
}

(Recibido el 29 de Octubre de 2020, Aceptado el 27 de Julio de 2020)

RESUMEN: La inclusión de novedosas tecnologías digitales en el mundo universitario requiere el análisis de sus ventajas e inconvenientes. Tanto para su empleo didáctico en la búsqueda de alcanzar distintos objetivos formativos como para el desarrollo de competencias digitales en el alumnado es necesario establecer experiencias que nos permitan obtener información para su correcto uso. Es el objetivo principal de esta investigación, que se fundamenta en un trabajo de campo inmersivo con realidad aumentada (RA) que tuvo como muestra a estudiantes universitarios a lo largo de dos cursos académicos. La metodología empleada en este trabajo exploratorio es descriptiva y cualitativa, para el que se han usado diferentes aplicaciones de RA. Como resultado principal se ha podido determinar que la integración de estas apps contribuye de manera significativa a la mejora de la competencia digital de los estudiantes universitarios a la vez que potencia la motivación en el aprendizaje. Sin embargo, se presenta como inconveniente principal su escasa presencia en las aulas y la necesidad de una formación previa del profesorado en su correcto empleo.

Palabras clave: realidad aumentada; realidad virtual; competencias digitales; motivación; formación del profesorado; educación superior.

\section{Augmented Reality (AR) and Training Processes at the University: Descriptive Study of New Applications for the Development of Digital Competences}

ABSTRACT: The inclusion of new digital technologies at university requires an analysis of their
advantages and disadvantages. It is necessary to establish experiences from two perspectives: didactic
use to achieve different training objectives and the development of digital competences in students, and 
to allow them to obtain information for their correct employment. This research is based on immersive fieldwork with Augmented Reality (AR) through a sample of university students over two academic years. The methodology used in this exploratory work is descriptive and qualitative, for which different AR applications have been used. As a main result, it has been determined that the integration of these apps contributes significantly to the improvement of digital competence of university students while enhancing motivation in learning. However, the main drawback comes from its low presence in the classroom and the need for training of teachers.

Keywords: Augmented Reality; Virtual Reality; Digital Competences; Motivation; Teacher Training; Higher Education.

Correspondencia: Eloy López-Meneses. Edificio 11, planta segunda, Despacho 25. Dpto. de Educación y Psicología Social. Facultad de Ciencias Sociales. Universidad Pablo de Olavide. Ctra. de Utrera, km. 1.41013 Sevilla. España. E-mail: evazquez@edu.uned.es

\section{Introducción}

Vivimos en una sociedad en la que las interacciones cotidianas se ven cada vez con mayor frecuencia condicionadas por las tecnologías de la información y la comunicación (TIC), y en la que el aprendizaje se concibe como una co-creación del conocimiento en comunidades potenciadas por la tecnología (Kali, Baram-Tsabari, y Schejter, 2019). En este sentido, vivimos una era de inmensos avances en las TIC que afectan todos los aspectos de nuestras vidas, y especialmente lo que sabemos y cómo aprendemos. En consecuencia, el imperativo tecnológico en educación se relaciona con una evolución y digitalización general de la sociedad y con la necesidad de nuevas competencias (Engen, 2019).

Hoy en día, la utilización pedagógica de las Tecnologías de la Información y la Comunicación (TIC) ha facilitado la creación compartida del conocimiento a través de comunidades de aprendizaje (Romero y Patiño, 2018) y un conjunto de beneficios y potencialidades con respecto a los métodos tradicionales de transmisión de contenidos (López-Belmonte et al., 2019; López-Meneses et. al, 2019). De igual manera, los cambios socioeducativos y tecnológicos del siglo XXI han contribuido a impulsar en las instituciones de Educación Superior profundas transformaciones orientadas al afianzamiento de nuevas tendencias que buscan favorecer la movilidad de estudiantes, graduados y personal docente, entre otros aspectos, con la finalidad de dar paso a una sociedad competitiva basada en el conocimiento y en las nuevas tendencias tecnológicas, donde los estudiantes se convierten en actores fundamentales para el logro de este propósito (Vázquez-Cano et al., 2015; Veytia, Gómez-Galán, y Cevallos, 2019). A su vez, como señalan Delgado-Vázquez, et al. (2019), estas desempeñan un papel de una enorme riqueza y los profesionales de la educación no pueden permanecer al margen.

En la misma línea de reflexión, el conocimiento y las habilidades con el uso de la tecnología son una parte esencial de la vida moderna (Juhaňák et al., 2019; Matosas-López et al., 2019). Además, estas tecnologías tienen el potencial de preparar a los estudiantes para la vida en el siglo XXI. A través del aprendizaje de estas nuevas habilidades, los estudiantes están preparados para afrontar los retos futuros que deben basarse en una comprensión adecuada de su mundo (Grimus, 2000). Bransford, Brown y Cocking (2000) creen que el uso de las TIC puede ayudar a los estudiantes a desarrollar las competencias necesarias para la globalización actual. Esto se debe a que 
pueden ayudar a los estudiantes a desarrollar sus habilidades, aumentar su motivación y ampliar sus conocimientos e información (Grabe y Grabe, 2007; Hussain et al., 2011). Por ello, uno de los principales planteamientos del proceso educativo es centrarse en el aprendizaje y en el estudiante desde un enfoque educativo basado en competencias digitales, con la convicción de que esto contribuirá a mejorar los procesos formativos universitarios. Del mismo modo, se puede inferir que los planes de estudios conducentes a la obtención de un título deberán, por tanto, tener entre sus objetivos la adquisición de ellas por parte del estudiantado, así como en los procedimientos para evaluar su adquisición.

Hablar de "competencias" es hacer mención a un concepto polisémico caracterizado no sólo por la diversidad de acepciones semánticas que se le han ido atribuyendo a lo largo del tiempo, sino que también por los usos que a este concepto se le ha ido dando en diferentes escenarios formativos y su vinculación con otros términos como estrategia, habilidad, etc., que hacen que la idea en si misma quede difusa (Cebrián y Junyent, 2015; Vázquez et al., 2017).

Diferentes autores indican que una competencia es un proceso en el cual las personas pueden resolver creativamente problemas, realizar actividades, formular preguntas, buscar información relevante, analizar, comprender y reflexionar al aplicar su conocimiento dando una respuesta a las demandas de un entorno real (Serrano, Biedermann, y Santolaya, 2016; Ramos, Chiva, y Gómez, 2017). A su vez, se puede entender por competencia digital la capacitación de saber utilizar la tecnología de manera eficaz para mejorar todas las áreas de nuestra vida diaria.

Ahora bien, la competencia digital no es una habilidad independiente, sino que toda ella supone un compendio de destrezas, habilidades y actitudes ante diferentes áreas y dimensiones de conocimiento (Rodríguez-García et al., 2019), donde el protagonista de la acción educativa es el estudiantado, que por su parte, han de hacer frente a toda esta sociedad tecnológica y que ha transformado las diferentes formas de comunicarse, aprender, acceder al trabajo, etc., en definitiva, vivir el presente y estar preparado para futuro (Gisbert y Lázaro, 2015).

Desde el ámbito formativo están surgiendo un amplio espectro de tecnologías emergentes, que ayudan a desarrollar las competencias digitales, entre ellas, la Realidad Aumentada (RA). Esta es una de las tecnologías, dentro del conjunto de la Realidad Virtual (RV) y lo que esta aporta en escenarios didácticos, que puede ofrecer un mayor impacto en la educación (Gómez-Galán, 2001, 2002 y 2014; Cabero, García-Jiménez y Barroso, 2016; Hood, 2017; Moreno-Martínez y Leiva, 2017; Fernández-Robles, 2018; Cabero et al., 2019), especialmente en la Educación Superior (Chang, Wu, y Hsu, 2013; Cuendet, et al., 2013; Cabero y García, 2016; Barroso y Gallego-Pérez, 2017) y está creciendo en los últimos años gracias a los dispositivos móviles, facilitando su acceso a todo el público (Cabero y Barroso, 2018).

Diferentes autores (Johnson y Adams, 2016; Cabero et al., 2019) expresan que la RA es el entorno en el que tiene lugar la integración de lo virtual y lo real, es decir, la combinación de información digital e información física en tiempo real a través de distintos dispositivos tecnológicos. Esta tecnología permite también mostrar información proporcionando materiales multimedia o textos vinculados a objetos o lugares, de forma sencilla e inmediata (Billinghurst, Kato, y Poupyrev, 2001) y abordar la percepción desde un enfoque innovador desde una capa visual añadida al entorno real (Maquilón, Mirete, y Avilés, 2017). La RA ofrece numerosas posibilidades educativas y un inmenso potencial para mejorar el aprendizaje y la enseñanza (Dunleavy, Dede, y Mitchell, 2009; Bacca et al., 2014; Prendes, 2015, Cabero et al., 2016). Además, proporciona 
a los usuarios el acceso a un contenido multimedia rico, variado y significativo, facilitándole un contexto relevante y con el que poder interactuar de manera inmediata (Han et al., 2015; Cabero y García, 2016).

Por ejemplo, la RA ayuda a los estudiantes a realizar exploraciones en el mundo real, al mostrar elementos virtuales junto a objetos reales facilita la observación de eventos que no pueden ser fácilmente observados a simple vista (Wu et al., 2013). De esta manera, aumenta la motivación de los estudiantes y les ayuda a adquirir mejores habilidades de investigación (Sotiriou y Bogner, 2008). Según Dunleavy, Dede y Mitchell (2009), la ventaja más significativa de RA es su capacidad única para crear entornos de aprendizaje híbridos e inmersivos que combinan objetos digitales y físicos, facilitando así el desarrollo de habilidades de procesamiento como el pensamiento crítico, la resolución de problemas y la comunicación a través de ejercicios de colaboración interdependientes. En este sentido, ayuda a la activación de procesos cognitivos de aprendizaje, el desarrollo de habilidades cognitivas y espaciales en los estudiantes, indistintamente de su edad y nivel académico, así como favorecer escenarios formativos más motivadores, colaborativos e interactivos (Cabero et al., 2016). Por último, diversos autores (Fombona Cadavieco y Vázquez-Cano, 2017; Fombona, Pascual, y Vázquez-Cano, 2020; Cabero y García 2016) indican entre sus limitaciones la falta de capacitación del docente y la escasez de recursos y objetivos de aprendizaje centrados en la RA.

Publicaciones recientes, como las de Bacca et al. (2014), Billinghurst et al. (2015), Chen et al. (2017), Diegmann et al. (2015), Dunleavy y Dede (2014), Palmarini et al., (2018) y Wu et al. (2013), han mostrado que la investigación en RA abarca un amplio espectro de objetivos y métodos. A su vez, Estados Unidos, Reino Unido y China son los países que abordan el estudio de la realidad virtual en la educación con un mayor número de publicaciones científicas (Liu et al., 2017).

Por otra parte, diversos estudios revelaron que el uso de la RA en la educación formal podría permitir a los educadores combinar esas aplicaciones con diversos enfoques pedagógicos, como el aprendizaje situado (Chang y Jen-ch'iang, 2013; Crandall et al., 2015), el aprendizaje basado en la investigación (Wang et al., 2014; Bressler y Bodzin, 2013; Chang, Wu y Hsu, 2013), y el aprendizaje basado en el juego (Hwang et al. 2015; Lu y Liu, 2015; Bressler y Bodzin, 2013). La RA se utiliza hoy en día en todos los niveles de enseñanza, desde el K-12 (Chiang, Yang y Hwang, 2014; Kerawalla et al., 2006) hasta el nivel universitario (Ferrer-Torregrosa et al., 2015).

\section{Objetivos y contexto de la investigación}

La experiencia innovadora inmersiva que constituye la base de esta investigación se desarrolló durante dos cursos académicos (2016-18), a través de tres sesiones de dos horas de duración cada una en las clases de Enseñanzas Prácticas y de Desarrollo (EPD) realizadas durante los meses de enero y febrero en la asignatura "TIC y Educación", correspondiente al primer año de la titulación del Grado de Educación Social y Doble Grado de Educación Social y Trabajo Social de la Facultad de Ciencias Sociales de la Universidad Pablo de Olavide, de Sevilla (España) con una carga de 7,3 Créditos ECTS (European Credit Transfer System). Los objetivos perseguidos son: (1) conocer las nuevas aplicaciones de RA en escenarios formativos y sus posibilidades pedagógicas en contextos educativos; (2) generar en el estudiantado actitudes proactivas ante las 
tecnologías aumentadas, y (3) desarrollar en el alumnado competencias de uso didáctico de las aplicaciones de RA.

\section{Referente a las apps de RA utilizadas en las sesiones prácticas son las siguientes:}

- Quiver: aplicación basada en la realidad aumentada y la virtualidad, consiste en colorear láminas impresas que se obtienen de la web: http://quivervision.com y posteriormente, con cualquier dispositivo móvil a través de su cámara, dichas láminas coloreadas adquieren vida generando escenarios aumentativos para mejorar los procesos de aprendizaje (Figura 1).

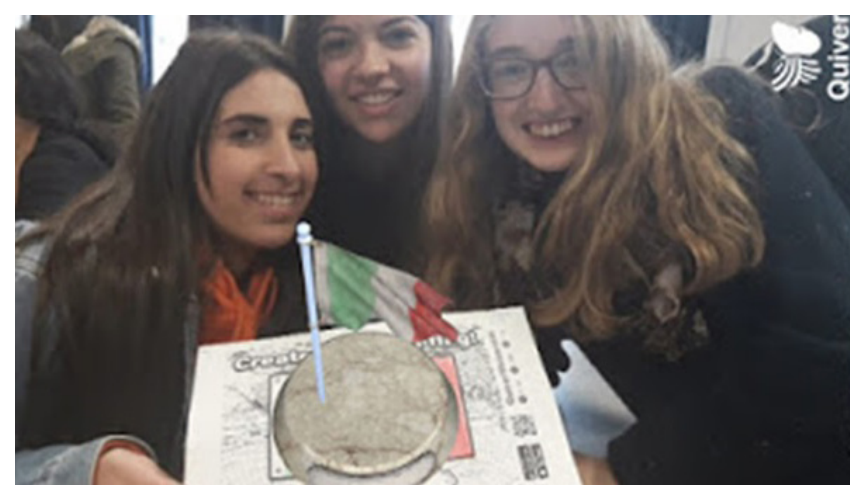

Figura 1. Quiver augmented reality. Fuente: Universidad Pablo de Olavide. Elaboración propia

- Chromville: es una aplicación similar a la app de Quiver siguiendo la misma dinámica de láminas basada en tecnología inmersiva. Las láminas impresas para colorear que actúan como marcadores para la génesis de entornos de fantasía aumentada a través de la cámara del dispositivo, se obtienen a través de esta web: https://chromville.com (Figura 2).

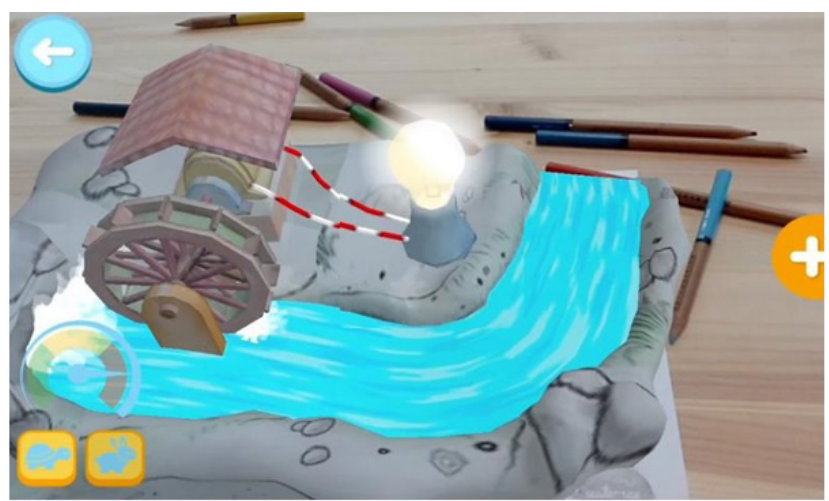

Figura 2. Chromville augmented reality. Fuente: Imascono. https://vimeo.com/166031138 
- Zookazam: a través de esta aplicación se puede añadir un amplio repertorio de animales de diversas especies en nuestro entorno real haciendo posible la recreación de escenas de fábulas. Es una app como las anteriores, disponible en AppStore y GooglePlay. Más información acerca de esta aplicación: http://www.zookazam.com (Figura 3).

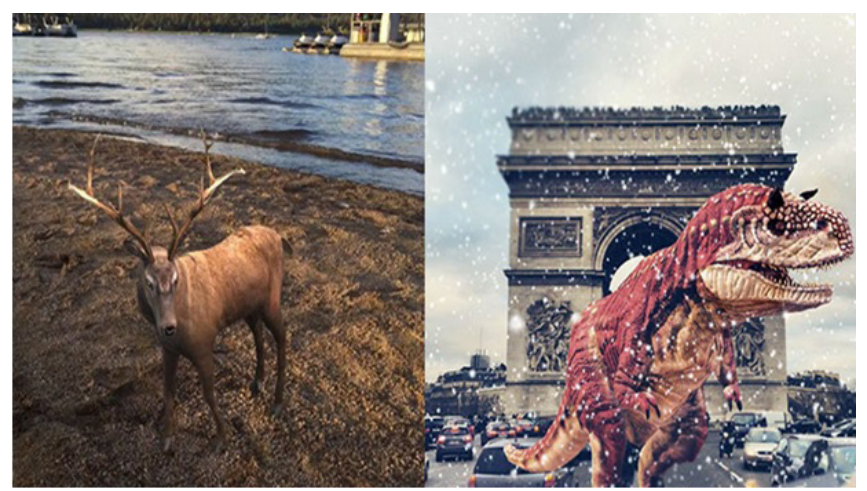

Figura 3. Zookazam augmented reality. Fuente: Zookazam. http://www.zookazam.com/

- Anatomy 4D. Con esta app de RA el estudiante puede conocer el interior del cuerpo humano y efectuar un viaje virtual para estudiar órganos, aparatos y sistemas del cuerpo humano. https://daqri.com/ (Figura 4).

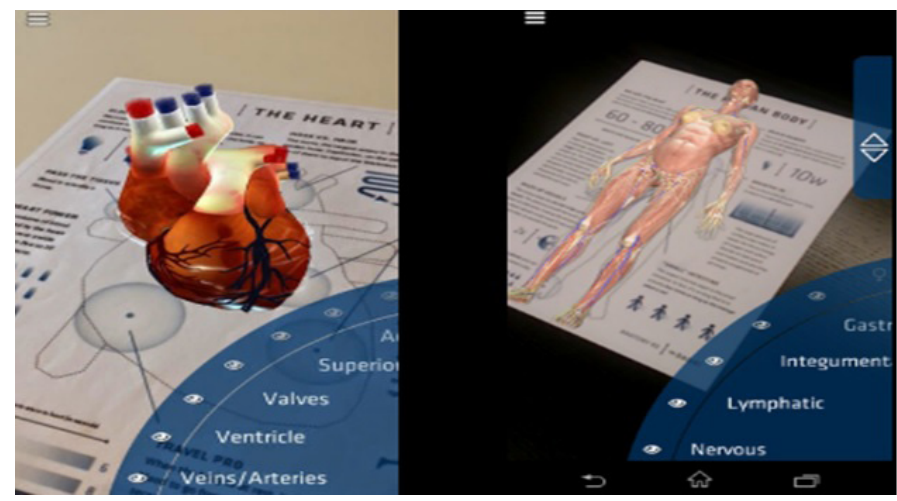

Figura 4. Anatomy4D augmented reality. Fuente: Citecmat. http://citecmat.blogspot.com/2014/12/anatomy-4d.html

\section{Metodología}

Se aborda un estudio exploratorio de corte cualitativo y descriptivo. Para ello, se parte de una concepción metodológica basada en la investigación-acción colaborativa virtual por parte del estudiante universitario como una manera de fomentar sus propias competencias genéricas y específicas en el Espacio Europeo de Educación Superior (Pool-Cibrian y Martínez-Guerrero, 2013). El objetivo principal consiste en conocer la percepción educativa de los estudiantes universitarios sobre la funionalidad de las apps educativas de RA que se han empleado como recurso didáctico durante dos cursos académicos 2016/18 en la asignatura: "Tecnologías de la Informa- 
ción y la Comunicación en Educación Social" de las titulaciones de Grado de Educación Social y Doble grado en Trabajo Social y Educación Social.

El análisis cualitativo se ha fundamentado en un proceso de codificación y categorización estructurado en dos etapas: la etapa descriptiva y la etapa interpretativa. El procedimiento se organizó en tres fases: 1) Fase 1: "Segmentación e identificación de unidades de significado y agrupación en categorías descriptivas". 2) Fase 2: "Construcción de un sistema de núcleos temáticos emergentes o metacategorías" y 3) Fase 3: "Identificación de dominios cualitativos (análisis secuencial y transversal de las metacategorías)".

La muestra participante estuvo compuesta por un total de 227 estudiantes cuya distribución se muestra en la Tabla 1.

Tabla 1. Muestra particpante.

\begin{tabular}{lcccc}
\hline & $\begin{array}{c}2016 / 17 \\
\text { Hombres }\end{array}$ & $\begin{array}{c}2016 / 17 \\
\text { Mujeres }\end{array}$ & $\begin{array}{c}2017 / 18 \\
\text { Hombres }\end{array}$ & $\begin{array}{c}2017 / 18 \\
\text { Mujeres }\end{array}$ \\
\hline $\begin{array}{l}\text { Primer curso } \\
\text { Doble Grado en Trabajo Social y Educación Social }\end{array}$ & 8 & 58 & 5 & 43 \\
$\begin{array}{l}\text { Primer curso } \\
\text { Grado en Educación Social }\end{array}$ & 6 & 51 & 4 & 52 \\
\hline
\end{tabular}

Por último, a finales del mes de febrero, los estudiantes implementaron el cuestionario "Uso didáctico de la RA" (Su enlace es: https://goo.gl/forms/STik3sI9KdPzZi773). El diseño del cuestionario partió de las consideraciones teóricas realizadas por Barroso y Gallego-Pérez (2017) y se revisó por juicio de expertos en dos rondas mediante estudio Delphi. Entre los objetivos del cuestionario estaba recabar las percepciones de los estudiantes en relación con las diferentes apps de RA más motivadora y útil utilizada en el Seminario de innovación tecnológica. En concreto, en nuestro estudio se analizaron las apreciaciones e impresiones de los estudiantes de las dos titulaciones relacionadas con las apps de RA empleadas en las aulas universitarias.

\section{Resultados}

El análisis e interpretación de las 227 aportaciones vertidas por el estudiantado muestran que las app más funcionales han sido: Quiver (45\%); Zookazam (23\%), Chromville (17\%) y, en último lugar, con un 15\% la app de RA relacionada con el cuerpo humano Antomy 4D. En cuanto a su fundamentación teórica, la mayoría del estudiantado informó que la app Quiver era la más interesante desde un punto de vista didáctico por ser muy intuitiva, fácil de manejar y muy adecuada para utilizarla en edades muy tempranas permitiendo colorear las láminas y luego convertirse en objetos animados. Y, en segundo lugar, la aplicación Zookazam por ser muy usable, rápida y permite un aprendizaje eficaz de diversos animales.

Referente a los estudiantes de Grado de Educación Social (2016-17) estimaron que las apps de RA más motivadoras utilizadas en el Seminario de Tecnologías Emergentes eran: Quiver con un 44\%, seguida de Zookazam (23), Anatomy 4D (19\%) y Chromville (14\%). Por último, 
expresaron que las apps de Quiver y Zookazam eran las más motivadora y educativas al ser muy fáciles de utilizar, usables, amigables y orientadas a los procesos educativo.

En cuanto al estudiantado de Doble Grado en Trabajo Social y Educación Social del curso académico 2017-18, se obtienen resultados semejantes a los obtenidos por el alumnado del curso académico anterior, en concreto, Quiver (45\%); Zookazam (24\%). En este curso los estudiantes también consideran interesante desde un punto de vista didáctico la app Anatomy 4D (17\%) seguida de la app Chromville (14\%). Referente a la titulación de Grado de Educación Social (201718) los estudiantes estimaban que la app de RA Quiver (43\%) junto con Zookazam (21\%) son las de mayor interés en el ámbito socio-educativo por su usabilidad y su aplicabilidad a contextos de colectivos infantiles.

Se presentan en la Figura 5 los resultados de frecuencias de los dos cursos académicos para observar su oscilación referente a las apps de RA más motivadoras y útiles para los procesos formativos utilizadas en el Seminario de tecnologías emergentes.

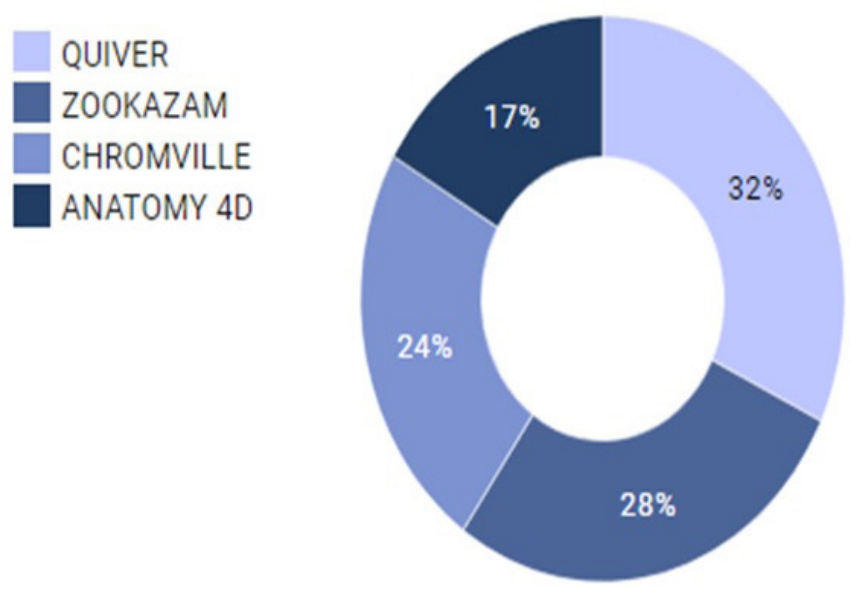

2016/2017

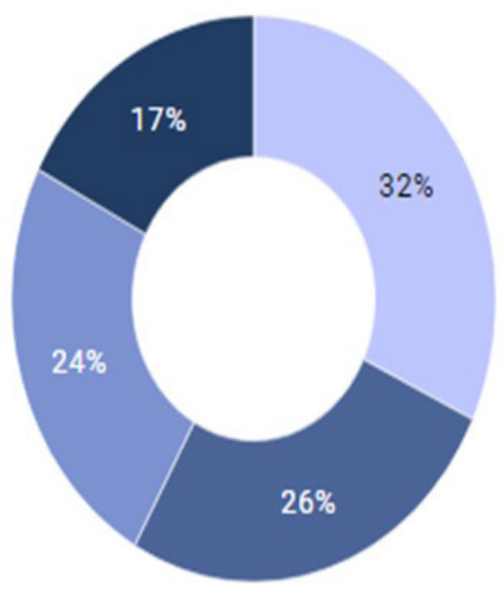

$2017 / 2018$

Figura 5. Comparativa de las frecuencias de los cursos académicos 2016/17 y 2017/18.

Fuente: Análisis estadístico del estudio. Elaboración propia.

Se puede inferir que el estudiantado de los dos cursos académicos (2016-18) aprecian que las aplicaciones de RA: Quiver y Zookazam utilizadas en el Seminario de tecnologías emergentes de la asignatura: "TIC y Educación Social”, son las más valiosas por su usabilidad, fácil manejo y por ser aplicaciones que ayudan a adquirir nuevos conocimientos en etapas educativas tempranas. No obstante, todas las aplicaciones empleadas han contado con la satisfacción de los estudiantes para su empleo.

\section{Discusión y conclusiones}

En una primera instancia, con los resultados obtenidos se constata en concordancia con otros estudios que cuando se incorpora a la enseñanza estas tecnologías inmersivas. Los estudiantes valoran muy positivamente la integración didáctica y las posibilidades de aplicación socio-profesional de estas experiencias educativas (Furió, Seguí y Vivó, 2015; Bicen y Bal, 2016; Cabero, Vázquez-Cano y López-Meneses, 2018, Cabero et al., 2019). Asimismo, perciben que se encuen- 
tran más motivados hacia el aprendizaje a través de la simulación que propicia esta tecnología (Bressler y Bodzin, 2013; Fombona y Vázquez-Cano, 2017) y mejoran sus resultados académicos (Kamarainen et al., 2013; Lu y Liu, 2015).

Asimismo, estimula un entorno proactivo de enseñanza (Cabero, Vázquez-Cano, y López Meneses, 2018; Fombona Cadavieco, Vázquez-Cano, y Del Valle Mejías, 2018; Cabero, Vázquez-Cano, López Meneses, y Jaén Martínez, 2020) y favorece un elevado grado de satisfacción en los alumnos (Han et al., 2015; Kim, Hwang y Zo, 2016). Con respecto a los aspectos más positivos del empleo didáctico dela RA, se ha evidenciado que favorece la construcción de competencias emergentes conducentes a mejorar capacidad de trabajo en equipo, el descubrimiento de nuevos recursos didácticos inmersivos útiles en los escenarios de la Educación Social y el Trabajo Social, desconocidos por la mayoría del estudiantado, lo cual puede ayudar al desarrollo de nuevos procesos formativos desde una perspectiva indagadora, constructivista y ubicua (Cabero, Vázquez-Cano y López-Meneses, 2018). Asimismo, los resultados de esta investigación corroboran otros estudios que muestran que esta tecnología emergente propicia el aprendizaje autónomo y aumenta la motivación de los estudiantes (Martín-Gutiérrez et al., 2015) y mejora con respecto a las enseñanzas tradicionales en aspectos de implicación y motivación (Di Serio, Ibáñez y Kloos, 2013), aportando contenidos que facilitan la contextualización del aprendizaje.

El análisis de la experiencia didáctica de aplicación de la RA con alumnado universitario nos permite aseverar como primera conclusión que la utilización de objetos con base en la tecnología aumentada en la Educación Superior, despierta verdadero interés entre los estudiantes. Este extremo también ha sido corroborado por diferentes estudios que muestran alto niveles de satisfacción cuando el alumnado utiliza esta tecnología y el aumento significativo de los niveles de motivación cuando los estudiantes están inmersos en acciones formativas con RA (Chin-Ming y Yen-Nung, 2011; Neven, Hala y Mohamed, 2011; Di Serio et al. 2013; Wojciechowski y Cellary, 2013; Kim, Hwang y Zo, 2016).

En concordancia con Fernández-Robles (2018), la realidad aumentada se puede presentar como una tecnología de verdadera utilidad para la formación universitaria, ya que permite trabajar con metodologías activas que ofrecen la posibilidad de visualizar el objeto desde diferentes perspectivas, facilita la adquisición de conocimientos de difícil acceso, posibilita la presentación de escenarios simulados, y enriquece el material impreso.

Asimismo, la percepción del alumnado de Educación Superior en las áreas de Ciencias Sociales de este estudio ha evidenciado que el empleo de actividades con base en la RA posibilita una mayor motivación y reflexión e incrementa la actitud positiva para aprender los contenidos de la asignatura. En este sentido, corrobora lo ya obtenido por Chang et al. (2013).

Por otra parte, como también se infiere del análisis de los resultados por parte de los estudiantes, resulta interesante ver cómo la RA es aplicable no solo en el proceso de enseñanza-aprendizaje, sino como un recurso útil y con gran potencial en el desempeño de sus competencias profesionales como educadores y trabajadores sociales. Así, con respecto a su aplicación en el ámbito profesional del educador y trabajador social, los estudiantes participantes han reseñado que la RA puede resultar de especial interés para el tratamiento de problemas de drogodependencia y su prevención entre adolescentes, la discapacidad asociada a la demencia en personas mayores y la faceta educativa en la infancia y la educación de menores (adolescentes) en la enseñanza formal y no formal. 
La aplicación de esta tecnología en el tratamiento psicosocial de estos problemas podría mejorar la adquisición de competencias por parte de los profesionales y, asimismo, servir de apoyo y mejora de las condiciones y tratamiento de los aspectos reseñados en los diferentes colectivos sociales. Además, el empleo de la RA ha sido útil en la construcción de competencias emergentes sobre utilización de TIC, capacidad de trabajo en equipo, el descubrimiento de nuevos recursos didácticos inmersivos útiles en los escenarios de la Educación Social y el Trabajo Social, desconocidos por la mayoría del estudiantado, lo cual puede ayudar al desarrollo de nuevos procesos formativos desde una perspectiva indagadora, constructivista y ubicua.

En este sentido, queremos destacar que las actividades didácticas con base en la RA pueden ser especialmente útiles en estudios preuniversitarios y de enseñanza superior relacionados con las áreas de Humanidades y Ciencias Sociales ya que permite un acceso a contenidos, muchas veces presentados de forma más unidireccional mediante medios impresos que no incrementan la motivación o requieren un mayor esfuerzo de abstracción entre los estudiantes. Con este recurso, los contenidos se visualizan de una forma más creativa, dinámica y real consiguiendo una didáctica más atractiva, innovadora y motivadora en cualquier nivel educativo.

A pesar de estos supuestos beneficios, creemos que se debe ahondar en las propuestas y modelos de integración de la RA teniendo en cuenta que su desarrollo debe contextualizarse debidamente; tomando en consideración los temas y recursos disponibles y cuál es la formación del profesorado; ya que, sin la adecuada formación, su uso puede llegar a resultar contraproducente pudiendo generar un retraso en el desarrollo de competencias y contenidos en la programación de la enseñanza. Además, hay que tener muy presentes los recursos con los que cuentan las instituciones educativas y los propios estudiantes para no generar una mayor brecha digital o discriminación por motivos económicos o de acceso a la tecnología. Asimismo, hay que garantizar que esta tecnología incorpore mecanismos de usabilidad y adaptabilidad a las diferentes necesidades especiales que puedan tener los estudiantes en las aulas (Yang, 2006; Chu et al., 2010; Hung et al., 2013).

Asimismo, experiencias universitarias de este tipo ayudan a expandir el modelo teórico didáctico conocido como Technology, Pedagogy, Content and Space (TPeCS) (Kali et al., 2019), TPeCS sostiene que una buena enseñanza hoy en día requiere no sólo "una comprensión de cómo la tecnología se relaciona con la pedagogía y el contenido" (como en el TPaCK de Mishra y Kohler, 2006, p. 1026), sino también una comprensión y habilidad para adaptar los espacios físicos existentes, aprovechar los espacios alternativos o diseñar otros nuevos.

Esto puede incluir el uso de tecnologías digitales como dispositivos móviles, realidad aumentada, espacios de fabricantes y, como punto focal para este estudio, pantallas digitales de gran tamaño (Tissenbaum y Slotta, 2019). Como apuntan Cabero y Martínez (2019) es el momento de comenzar la transformación de la alfabetización digital a la competencia digital. Su adquisición debe efectuarse a través de las prácticas de la formación inicial y promocionar nuevos escenarios formativos donde el estudiante se convierta en "prosumidor" de mensajes tecnológicos.

En definitiva, se puede considera que el desafío para las universidades, en este marco, reside en rediseñar sus matrices formativas alrededor de las competencias profesionales más que alrededor de las tradicionales asignaturas de forma que se potencie el desarrollo de propuestas didácticas que involucren el trabajo colaborativo para el fomento de un aprendizaje significativo (Mendieta, Cobos y Vázquez-Cano, 2016) y el incremento progresivo en la actividad docente 
de la utilización de los recursos educativos electrónicos (Vázquez-Cano, López Meneses y Sánchez-Serrano, 2015; Infante-Moro et al., 2017; Cabero, Vázquez-Cano, y López-Meneses, 2018). En última instancia, en concordancia con Cabero et al. (2015) la realidad aumentada puede ser una interesante tendencia emergente tecno social eficiente en contextos formativos y ayudar a la génesis de procesos de innovación curricular en las aulas aumentadas.

\section{Agradecimientos}

Este estudio surge de la directiva del proyecto de innovación pedagógica "Formación didáctica en Cloud Computing": Competencias digitales, estrategias didácticas y e-actividades con tecnología Web 2.0 en el EEES”, en el marco de la Acción 2 de Proyectos de Innovación Pedagógica y Desarrollo Docente, financiado por el Rectorado de la Universidad Pablo Olavide de Sevilla, en el marco del grupo de investigación EduInnovagogía® (HUM-971).

\section{Referencias}

Bacca, J., Baldiris, S. Fabregat, R., Graf, S., y Kinshuk, J. (2014). Augmented Reality Trends in Education: A Systematic Review of Research and Applications, Educational Technology \& Society, 17(4), 133-149.

Barroso, J. y Gallego-Pérez, O. M. (2017). Producción de recursos de aprendizaje apoyados en Realidad Aumentada por parte de estudiantes de magisterio. Revista de Educación Mediática y TIC (Edmetic), 6(1), 23-38.

Bicen, H. y Bal, E. (2016). Determination of student opinions in augmented reality. World Journal on Educational Technology: Current Issues, 8(3), 205-209.

Billinghurst, M., Clark, A., y Lee, G. (2015). A survey of augmented reality. Foundations and Trends in Human. Computer Interaction, 8(2-3), 73-272.

Billinghurst, M., Kato, H. y Poupyrev, I. (2001). The magicbook-moving seamlessly between reality and virtuality. IEEE Computer Graphics and Applications, 21, 6-8.

Blas Padilla, D., Vázquez-Cano, E., Morales Cevallos, M., \& López Meneses, E. (2019). Uso de apps de realidad aumentada en las aulas universitarias. Campus virtuales, 8(1), 37-48.

Bressler, D. M. y Bodzin, A. M. (2013). A mixed methods assessment of students'flow experiences during a mobile augmented reality science game. Journal of Computer Assisted Learning, 29(6), 505-517.

Cabero, J., Vázquez-Cano, E., López Meneses, E., y Jaén Martínez, A. (2020). Posibilidades formativas de la tecnología aumentada. Un estudio diacrónico en escenarios universitarios. Revista Complutense de Educación, 31(2), 143-154. https://dx.doi.org/10.5209/rced.61934

Cabero, J., Leiva Olivencia, J. J., Moreno Martínez, N. M., Barroso Osuna, J., y López Meneses, E. (2016). Realidad aumentada y educación. Innovación en contextos formativos. Barcelona: Octaedro.

Cabero J., Leiva J. J., Moreno N. M., Barroso J., y López-Meneses, E. (2016). Realidad Aumentada y Educación. Innovación en contextos formativos. Barcelona: Octaedro.

Cabero, J. y Barroso, J. (2018). Los escenarios tecnológicos en Realidad Aumentada (RA): posibilidades educativas en estudios universitarios. Aula Abierta, 47(3), 327-336. 
Cabero, J. y García, F. (Coords.) (2016). Realidad aumentada. Tecnología para la formación. Madrid: Síntesis.

Cabero, J. y Martínez, A. (2019). Las Tecnologías de la Información y Comunicación y la formación inicial de los docentes. Modelos y competencias digitales. Profesorado. Revista de Currículum y Formación de Profesorado, 23(3), 247-268. doi:10.30827/profesorado. v23i3.9421

Cabero, J., Barroso, J., Llorente, C. y Fernández-Martínez, Mª M. (2019). Educational Uses of Augmented Reality (AR): Experiences in Educational Science. Sustainability, 11, 4990; doi:10.3390/su11184990

Cabero, J., García-Jiménez, F., y Barroso, J. (2016). La producción de objetos de aprendizaje en "Realidad Aumentada": la experiencia del SAV de la Universidad de Sevilla. International Journal of Educational Research and Innovation (IJERI), 6, 110-123

Cabero, J., Vázquez-Cano, E., y López Meneses, E. (2018). Uso de la realidad aumentada como recurso didáctico en la enseñanza universitaria. Formación Universitaria, 11(1) 25-34. doi: http://dx.doi.org/10.4067/S0718-50062018000100025

Cabero, J., y Barroso, J. (2018). Los escenarios tecnológicos en Realidad Aumentada (RA): posibilidades educativas en estudios universitarios. Aula Abierta, 47(3), 327-336.

Cabero, J. y García, F. (Coords.) (2016). Realidad aumentada. Tecnología para la formación. Madrid: Síntesis.

Cebrián, G. y Junyent, M. (2015). Competencies in Education for sustainable development: Exploring the student teachers' view. Sustainability, 7(3), 2768-2786.

Chang, H., Wu, K., y Hsu, Y. (2013). Integrating a mobile augmented reality activity to contextualize student learning of a socioscientific issue. British Journal of Educational Technology, 44, 3, 95-99.

Chang, Y. H. y Jen-ch'iang, L. I. U. (2013). Applying an AR Technique to Enhance Situated Heritage Learning in a Ubiquitous Learning Environment. TOJET: The Turkish Online Journal of Educational Technology, 12(3), 21-32.

Chen, P., Liu, X., Cheng, W., y Huang, R. (2017). A review of using Augmented Reality in Education from 2011 to 2016. In Innovations in Smart Learning (pp. 13-18). Springer Singapore.

Chiang, T. H., Yang, S. J., y Hwang, G.-J. (2014). Students' online interactive patterns in augmented reality-based inquiry activities. Computers \& Education, 78, 97-108.

Chin-Ming, C.; Yen-Nung, T. (2011). Interactive augmented reality system for enhancing library instruction in elementary schools. Computers \& Education, 59, 638-652.

Chu, H. C., Hwang, G. J., y Tsai, C. C. (2010). A knowledge engineering approach to developing mindtools for context-aware ubiquitous learning. Computers \& Education, 54(1), 289-297.

Crandall, P. G., Engler, R. K., Beck, D. E., Killian, S. A., O’Bryan, C. A., Jarvis, N., y Clausen, E. (2015). Development of an Augmented Reality Game to Teach Abstract Concepts in Food Chemistry. Journal of Food Science Education, 14(1), 18-23.

Cuendet, S., Bonnard, Q., Do-Lenh, S., y Dillenbourg, P. (2013). Designing augmented reality for the classroom. Computers \& Education, 68, 557-569.

Delgado-Vázquez, A. Vázquez-Cano, E., Belando-Montoro, M. R., y López-Meneses, E. (2019). Análisis bibliométrico del impacto de la investigación educativa en diversidad funcional y competencia digital: Web of Science y Scopus. Aula Abierta, 48(2), 147-156. 
Di Serio, A.; Ibáñez, M. B.; Delgado, C. (2013). Impact of an augmented reality system on students' motivation for a visual art course. Computers \& Education, 68, 586-596. doi: 10.1016/j. compedu.2012.03.002

Diegmann, P., Schmidt-Kraepelin, M., Van den Eynden, S., y Basten, D. (2015). Benefits of Augmented Reality in Educational Environments - A Systematic Literature Review. Wirtschaftsinformatik, 3(6), 1542-1556.

Dunleavy, M., Dede, C., y Mitchell, R. (2009). Affordances and limitations of immersive participatory augmented reality simulations for teaching and learning. Journal of Science Education and Technology, 18(1), 7-22. doi:10.1007/s10956-008-9119-1.

Dunleavy, M. y Dede, C. (2014). Augmented reality teaching and learning. In Handbook of research on educational communications and technology (pp. 735-745). Springer.

Engen, B. K. (2019). Understanding social and cultural aspects of teachers' digital competencies. Comunicar, 61, 9-19. doi: https://doi.org/10.3916/C61-2019-01

Fernández-Robles, B. (2018). La utilización de objetos de aprendizaje de realidad aumentada en la enseñanza universitaria de educación primaria. International Journal of Educational Research and Innovation (IJERI), 9, 90-104.

Ferrer-Torregrosa, J., Torralba, J., Jimenez, M., García, S., y Barcia, J. (2015). ARBOOK: Development and assessment of a tool based on augmented reality for anatomy. Journal of Science Education and Technology, 24(1), 119-124.

Fombona, J. y Vázquez-Cano, E. (2017). Posibilidades de utilización de la Geolocalización y Realidad Aumentada en el ámbito educativo. Educación XX1, 20(2), 319-342. doi: https:// doi.org/10.5944/educxx1.19046

Fombona, J., Vázquez-Cano, E., y Del Valle Mejías (2018). Análisis de la geolocalización y realidad aumentada en dispositivos móviles, propuestas socio-educativas relacionadas con el entorno y las salidas de campo. Profesorado. Revista de Currículum y formación del profesorado, 22(4), 197-222. doi:10.30827/profesorado.v22i4.8413

Fombona, J., Pascual, M. A., y Vázquez-Cano, E. (2020). Augmented Reality: a new way to build knowledge. Bibliometric analysis and apps testing. IEEE Revista Iberoamericana de Tecnologías del Aprendizaje, 15(1), 17-25 doi: 10.1109/RITA.2020.2979167

Furió, M. C. Seguí, J., y Vivó, R. (2015). Mobile learning vs. Traditional classroo lessons: a comparative study. Journal of Computer Assisted Learning, 31, 189-201.

Gisbert, M. y Lázaro, J. L. (2015). Professional development in teacher digital competence and improving school quality from the teachers' perspective: a case study. Journal of New Approaches in Educational Research, 4(2), 115-122. https://doi.org/10.7821/naer.2015.7.123

Gómez-Galán, J. (2001). Aplicaciones Didácticas y Educativas de las Tecnologías RIV (Realidad Infovirtual) en Entornos Telemáticos. En VV.AA. Actas del XIII Congreso Internacional de Ingeniería Gráfica: Eliminando Fronteras entre lo Real y lo Virtual. (pp. 29-43). Badajoz: AEIA-UEX.

Gómez-Galán, J. (2002). Education and Virtual Reality. En N. Mastorakis (ed.). Advances in Systems Engineering, Signal Processing and Communications. (pp. 345-350). WSEAS Press. Gómez-Galán, J. (2014). Realidad Virtual en la Arqueología y el Arte: Orientaciones Didácticas y Formativas. En VV.AA. Arte y Sociedad: Bellas Artes y Sociedad Digital. (pp. 5-17). Málaga: FUAIG. 
Grabe, M. y Grabe, C. (2007). Integrating technology for meaningful learning (5th ed.). Houghton Mifflin.

Grimus, M. (2000). ICT and multimedia in the primary school. In 16th conference on educational uses of information and communication technologies, Beijing, China.

Han, J., Jo, M., Hyun, E., y So, H. (2015). Examining young children's perception toward augmented reality-infused dramatic play. Education Technology Research Development, 63, 455-474.

Hood, K. (2017). Telling Active Learning Pedagogies Apart: from theory to practice. Journal of new Approaches in Educational Research, 6(2), 144-152. doi: http://dx.doi.org/10.7821/ naer.2017.7.237

Hung, P. H., Hwang, G. J., Lin, Y. F., Wu, T. H., y Su, I. H. (2013). Seamless connection between learning and assessmentapplying progressive learning tasks in mobile ecology inquiry. Educational Technology \& Society, 16(1), 194-205.

Hussain, A. J., Morgan, S., y Al-Jumeily, D. (2011). How Does ICT Affect Teachings and Learning within School Education. In Developments in E-systems Engineering (DeSE), (pp. 250-254). IEEE.

Hwang, G. J., Wu, P. H., Chen, C. C., y Tu, N. T. (2015). Effects of an augmented reality-based educational game on students' learning achievements and attitudes in real-world observations. Interactive Learning Environments, 24(8), 1895-1906. doi: 10.1080/10494820.2015.1057747

Infante-Moro, A., Infante-Moro, J. C., Torres-Díaz, J. C., y Martínez-López, F. J. (2017). Los MOOC como sistema de aprendizaje en la Universidad de huelva (UhU). IJERI: International Journal of Educational Research and Innovation, 7, 13-24.

Johnson, L., Adams Becker, S., Cummins, M., Estrada, V., Freeman, A., y Hall, C. (2016). NMC Horizon Report: 2016 Higher Education Edition. Austin, Texas: The New Media Consortium.

Juhaňák, L., Zounek, J., Záleská, K., Bárta, O., y Vlčková, K. (2019). The relationship between the age at first computer use and students' perceived competence and autonomy in ICT usage: A mediation analysis. Computers \& Education, $141 \mathrm{https} / / \mathrm{doi} .0 \mathrm{rg} / 10.1016 / \mathrm{j}$. compedu.2019.103614.

Kali, Y., Baram-Tsabari, A., y Schejter A. (Eds.) (2019). Learning in a networked society: Spontaneous and designed technology enhanced learning communities. Springer's Computer Supported Collaborative Learning Series.

Kamarainen, A. A. M., Shari, M, Tina, G., Allison B., Diana, M, Shane, T., y Dede. C. (2013). EcoMOBILE: Integrating augmented reality and probeware with environmental education field trips. Computers \& Education 68, 545-556 doi:10.1016/j.compedu.2013.02.018.

Kerawalla, L., Luckin, R., Seljeflot, S., y Woolard, A. (2006). "Making it real": Exploring the potential of augmented reality for teaching primary school science. Virtual Reality, 10(3-4), 163-174.

Kim, K., Hwang, J., y Zo, H. (2016). Understanding users' continuance intention toward smartphone augmented reality applications. Information development, 32(2), 161-174.

Liu, D., Dede, C., Huang, R., y Richards, J. (2017). Virtual, Augmented, and Mixed Realities in Education. Springer.

López-Belmonte, J., Pozo, S., Morales-Cevallos, Ma . B., y López-Meneses, E. (2019). Competencia digital de futuros docentes para efectuar un proceso de enseñanza y aprendizaje mediante 
realidad virtual. EDUTEC. Revista Electrónica de Tecnología Educativa, 67, 1-15. https:// doi.org/10.21556/edutec.2019.67.1327

López-Belmonte, J., Pozo-Sánchez, S. y Fuentes-Cabrera, A. (2019). Recursos tecno-pedagógicos de apoyo a la docencia: La realidad aumentada como herramienta dinamizadora del profesor sustituto International Journal of Educational Research and Innovation (IJERI), 12, 122-136.

López-Meneses, E., Vázquez-Cano, E., Gómez-Galán, J., y Fernández-Márquez, E. (2019). Pedagogía de la innovación con tecnologías. Un estudio de caso en la Universidad Pablo de Olavide. El Guiniguada. Revista de Investigaciones y Experiencias en Ciencias de la Educación, 28, 76-92.

Lu, S. J. y Liu, Y. C. (2015). Integrating augmented reality technology to enhance children's learning in marine education. Environmental Education Research, 21(4), 525-541.

Maquilón, J. J., Mirete Ruiz, A. B., y Avilés Olmos, M. (2017). La realidad aumentada (R.A). Recursos y propuestas para la innovación educativa. Revista Electrónica Interuniversitaria de Formación del Profesorado, 20(2), 183-203.

Martín-Gutiérrez, J., Fabiani, P., Benesova, W., Meneses, M. D., y Mora, C. E. (2015). Augmented reality to promote collaborative and autonomous learning in higher education. Computers in Human Behavior, 51, 752-761. https://doi.org/10.1016/j.chb.2014.11.093

Matosas-López, L., Aguado-Franco, J. C., y Gómez-Galán, J. (2019). Constructing an instrument with behavioral scales to assess teaching quality in blended learning modalities. Journal of New Approaches in Educational Research, 8(2), 142-165. https://doi.org/10.7821/ naer.2019.7.410

Mendieta, C., Cobos, D., y Vázquez-Cano, E. (2016). La percepción de los docentes sobre la funcionalidad educo-formativa de las TIC en la Universidad nacional Autónoma de nicaragua (UNAM-Managua). Revista Latinoamericana de Tecnología Educativa (RELATEC), 15(3), 113-126

Mishra, P. y Koehler, M. J. (2006). Technological pedagogical content knowledge: A framework for teacher knowledge. Teachers college record, 108(6), 1017

Moreno-Martínez, N. y Leiva, J. J. (2017). Experiencias formativas de uso didáctico de la realidad aumentada con alumnado del grado de educación primaria en la universidad de Málaga. Revista de Educación Mediática y TIC (Edmetic), 6(1), 81-104.

Neven, A. M., Hala, H., y Mohamed, I. (2011). ARSC: Augmented Reality Student Card An Augmented reality Solution for the educational field. Computers \& Education, 56, 1045 1061. doi: 10.1016/j.compedu.2010.10.019.

Palmarini, R., Erkoyuncu, J. A., Roy, R., y Torabmostaedi, H. (2018). A systematic review of augmented reality applications in maintenance. Robotics and Computer-Integrated Manufacturing, 49, 215-228

Pool-Cibrian, W. J. y Martínez-Guerrero, J. I. (2013). Autoeficacia y uso de estrategias para el aprendizaje autorregulado en estudiantes universitarios. Revista Electrónica de Investigación Educativa, 15(3), 21-37.

Prendes, C. (2015). Realidad aumentada y educación: análisis de experiencias prácticas, Pixel-Bit. Revista de Medios y Educación, 46, 187-203. 
Ramos, G., Chiva, I., y Gómez, M. B. (2017). Las competencias básicas en la nueva generación de estudiantes universitarios: Una experiencia de Innovación. Revista de Docencia Universitaria, 15(1), 37-55. doi:10.4995/redu.2017.5909

Rodríguez-García, A. M., Raso-Sánchez, F., y Ruiz-Palmero, J. R. (2019). Competencia digital, educación superior y formación del profesorado: un estudio de meta-análisis en la Web of Science. Pixel-Bit. Revista de Medios y Educación, 54, 65-81. https://doi.org/10.12795/ pixelbit.2019.i54.04

Romero, M., y Patiño, A. (2018). Usos pedagógicos de las TIC: del consumo a la co-creación participativa. Revista Referencia Pedagógica, 6(1), 2-15.

Serrano, T. A., Biedermann, A. M., y Santolaya, S. J. (2016). Perfil, objetivos, competencias y expectativas de futuro profesional de los estudiantes del Grado en Ingeniería en Diseño Industrial y Desarrollo de Producto de la Universidad de Zaragoza. Revista de Docencia Universitaria, 14(1), 69-96. doi:10.4995/ redu.2016.5908

Sotiriou, S. y Bogner, F. X. (2008). Visualizing the invisible: Augmented reality as an innovative science education scheme. Advanced Science Letters, 1(1), 114-122.

Tissenbaum, M. y Slotta, J. D. (2019). Developing a smart classroom infrastructure to support real-time student collaboration and inquiry: a 4-year design study. Instructional Science, 47(4), 423-462 doi: https://doi.org/10.1007/s11251-019-09486-1

Vázquez-Cano, E., Reyes, M., Colmenares, L., y López-Meneses, E. (2017). Competencia digital del alumnado de la Universidad Católica de Santiago de Guayaquil. Revista opción, 83, 229-251.

Vázquez-Cano, E., López Meneses, E., y Sánchez-Serrano, J. L. (2015). Analysis of social worker and educator's areas of intervention through multimedia concept maps and online discussion forums higher Education. Electronic Journal of e-Learning, 13(5), 333-346

Veytia M. G., Gómez-Galán, J., y Cevallos, M. B. (2019). Competencias investigativas y mediación tecnológica en doctorandos de Iberoamérica. IJERI: International Journal of Educational Research and Innovation, 12, 1-19.

Wang, H. Y., Duh, H. B. L., Li, N., Lin, T. J., y Tsai, C. C. (2014). An investigation of university students' collaborative inquiry learning behaviours in an augmented reality simulation and a traditional simulation. Journal of Science Education and Technology, 23(5), 682-691.

Wojciechowski, R., y Cellary, W. (2013). Evaluation of learners' attitude toward learning in ARIES augmented reality environments. Computers \& Education, 68, 570-585. doi: 10.1016/j. compedu.2013.02.014

Wu, H. K., Lee, S. W. Y., Chang, H. Y., y Liang, J. C. (2013). Current status, opportunities and challenges of augmented reality in education. Computers \& Education, 62, 41-49.

Yang, S. J.H. (2006). Context aware ubiquitous learning environments for peer-to-peer collaborative learning. Educational Technology \& Society, 9(1), 188-201. 